\title{
Impact of simulated sunlight on the degradation of pendimethalin in surface water in a scale-up experiment in accordance to OECD TG 309
}

\author{
Dieter Hennecke ${ }^{1 *}\left(\mathbb{D}\right.$, Jan Hassink², Judith Klein ${ }^{1}$ and Mike Kruse ${ }^{1}$
}

\begin{abstract}
Background: OECD TG 309 "Aerobic Mineralisation in Surface Water" (OECD Test Guideline 309 (2004)) is currently used in regulation for the assessment of the persistence of chemicals in surface water. The test is performed in a batch under defined conditions. Processes which might be relevant for the fate of a chemical in surface water like direct and indirect photolysis are not addressed. In order to study the impact of sunlight on the fate in surface water, pendimethalin, a herbicidal active substance with known sensitivity to aqueous photolysis, was used. This was tested in an experiment based on OECD 309, but scaled up to $900 \mathrm{~L}$ size with a $1.40 \mathrm{~m}$ water column and irradiated with special lamps in order to monitor the fate of the substance at different water depths, in comparison to dark controls.

Results: Pendimethalin degraded significantly under the applied test conditions. The test setups with exposure to simulated sunlight showed a significant faster degradation compared to the dark experiments. Application of $900 \mathrm{~g}$ sediment ( $1 \%$ of test system) to the test system further increased the degradation rate. In the simulated sunlight setups a faster degradation was observed in the upper water layer, hence, the concentration of the photolysis products were highest in the upper water layer, where the highest light intensity was measured. With decreasing light intensity in deeper water layers photolysis products were still detected at a water depth of $125 \mathrm{~cm}$. Mineralisation up to $22 \%$ of the applied radioactivity was observed. In the dark controls neither significant amounts of photolysis products nor any mineralisation were detected. Among the different sampling depths no significant differences were observed. It was further observed that the dark controls became anaerobic at the end of the incubation time. This has been observed previously in standard laboratory tests according to OECD 309.
\end{abstract}

Conclusions: The study proves that for a substance sensitive to photolysis like pendimethalin, exposure to simulated sunlight leads to a significantly reduced degradation half-life $\left(\right.$ Deg $\left._{50}\right)$ in surface water compared to the dark control. In the standard test according to OECD guideline 309 the influence of photolytical degradation is not considered and thus the laboratory test can lead to the overestimation of the persistence of a chemical in surface water and consequently to a false-positive evaluation in the overall PBT assessment (ECHA Guidance on Information Requirements and Chemical Safety Assessment 2004), when only the results of the standard OECD 309 study are considered.

Keywords: OECD TG 309, Photolysis, Persistence, Lake simulation, Pendimethalin

\footnotetext{
*Correspondence: dieter.hennecke@ime.fraunhofer.de

${ }^{1}$ Fraunhofer Institute for Molecular Biology and Applied Ecology, Auf dem Aberg 1, 57392 Schmallenberg, Germany

Full list of author information is available at the end of the article
}

\section{Background}

OECD TG 309 "Aerobic Mineralisation in Surface Water" [1] is currently used under different regulatory frameworks for the assessment of the persistence of chemicals in surface water. The standard test is performed as a

\section{Springer Open}

(c) The Author(s) 2020. This article is licensed under a Creative Commons Attribution 4.0 International License, which permits use, sharing, adaptation, distribution and reproduction in any medium or format, as long as you give appropriate credit to the original author(s) and the source, provide a link to the Creative Commons licence, and indicate if changes were made. The images or other third party material in this article are included in the article's Creative Commons licence, unless indicated otherwise in a credit line to the material. If material is not included in the article's Creative Commons licence and your intended use is not permitted by statutory regulation or exceeds the permitted use, you will need to obtain permission directly from the copyright holder. To view a copy of this licence, visit http://creativeco mmons.org/licenses/by/4.0/. 
batch analysis to measure biodegradation under defined conditions. It is conducted with and without sediment but the effect of suspended solids on the degradation rate of chemicals in the test is unclear [2]. A major point of criticism is the missing guidance for a specific experimental setup. The current guideline allows too much variability of the experimental setup, which has a significant influence on the results [3]. As a consequence studies according to OECD 309 can be simple or very complex [2]. Further it was stated that an OECD 309 test is rather an expensive hydrolysis test and that substances that are not readily biodegradable are likely to fulfil the persistence criterion for water unless they are hydrolyzed [4].

Processes like direct and indirect photolysis, which might be relevant for the fate of a chemical in surface water, are not addressed. The guideline allows "diffuse light" but does not specify any concrete light conditions. Thus, most studies are conducted in the absence of light. Several publications prove the relevance of photolysis beside biodegradation in natural surface water for the fate of selected organic substances [5-7]. If the assessment of persistence [8] in surface water is triggered by the result of the OECD 309 study only, substances which are hydrolytically stable but sensitive to photolysis might be falsely assessed as persistent, not only for surface water but also within the overall PBT assessment according to ECHA [8].

For pesticide registration, direct photolysis studies are mandatory, indirect photolysis studies optional. However, the primary purpose of these tests is not to determine rate constants, which are considered later in the exposure assessment, but to get information on the occurrence of potentially stable transformation products. These would have to be considered for further risk assessment in registration approval processes.

Direct photolysis can be an important degradation process in the water phase. Only wavelengths of UV/visible light $(290-800 \mathrm{~nm})$ are considered in those tests as they represent the sunlight spectrum. As a consequence, chemicals that absorb light of this wavelength range can undergo direct photolysis in the water phase. Indirect photolysis in natural water is tested in the same way as direct photolysis, except that water from a natural system or natural water with nitrate or simulated natural water is used instead of a sterile aqueous buffer system [9]. In contrast to direct photolysis, indirect photolysis is governed initially by the spectroscopic properties of the natural water. In general, both indirect and direct photolysis can proceed simultaneously [10]. However, there is no requirement regarding the water quality parameters for testing indirect photolysis. Castro-Jiménez and Van de Meent [11] have reviewed the available literature on photolytic degradation of chemical substances under environmental conditions and concluded that light absorption in natural water is significantly lower than measured in laboratory water, with photodegradation about 30 times slower in typical fresh water. The relative importance of direct photolysis versus indirect photolysis varies and is dependent both on the substance and the media. Indirect photodegradation is stimulated in natural environmental waters by the presence of dissolved organic matter or nitrate which is not present in pure laboratory water. In natural waters, which have to be used for OECD 309, both processes can be relevant but in the standard OECD 309 setup the influence of light on the degradation of the test item is not considered.

In the case of plant protection products the exposure of a small freshwater body with sediment at the edge of an agricultural field via spray drift, run-off or drainage is highly relevant. Hence, beside the photolytical processes in the upper layer of a water body, there is a particular relevance to investigate as to which water depth direct and indirect photolysis might contribute to degradation since the light intensity decreases with water depth.

To study the influence of light on the degradation of a test substance in different water layers requires a modified test system. As a major modification the size of the test system had to be increased by orders of magnitude to be able to investigate processes in water depths below $1 \mathrm{~m}$. One further modification from OECD 309 is the static test performance, which is needed to analyse processes in different water layers. OECD 309 requires permanent mixing of the test vessels by shaking or stirring, but in the present study this would not comply with the study aim. Nevertheless, the test system should still follow the basic principles of OECD 309 with regard to test medium, test temperature, application rate, sampling and chemical analysis and maximum incubation time. A laboratory OECD 309 study with pendimethalin was already conducted and with a half-life of 193 days a poor degradation of pendimethalin was observed under standard conditions, including stirring [12].

Stainless steel containers with the shape of a vertically cut cylinder were filled with surface water taken from a natural lake and maintained at $20{ }^{\circ} \mathrm{C}$. Sampling of different water depths were performed without disturbing the water body significantly. Pelagic as well as suspended sediment setups were tested in parallel, whereby in the absence of mechanical mixing the suspended sediment settled within a few days at the bottom of the test vessel. To study the influence of light, a set of two test vessels were exposed to simulated sunlight while two further test vessels were incubated under dark conditions.

As test substance the herbicide pendimethalin (CAS No. 40487-42-1) was selected based on its substance intrinsic properties. Pendimethalin is hydrolytically 
stable but susceptible to aqueous photolysis with halflives of 1.5 to 5 days under continuous irradiation over a testing period of 15 days in a photolysis study according to OECD 316 [12]. In addition, pendimethalin has a low water solubility $\left(0.3 \mathrm{mg} / \mathrm{L}\right.$ at $\left.20^{\circ} \mathrm{C}\right)$ and a very high adsorption to organic matter with a mean $K_{\text {Foc }}$ value of $13,792 \mathrm{~mL} / \mathrm{g}$ [12]. In water/sediment systems pendimethalin rapidly partitions into the sediment [12]. The ${ }^{14} \mathrm{C}$-labelling of the test substance enables the determination of the degradation route as well as to establish a mass balance which is an important parameter in such a large size simulation system.

\section{Methods}

\section{Test and reference substances}

For the study a mixture of non radiolabeled pendimethalin (99.5\%, BASF SE) and ${ }^{14} \mathrm{C}$-radiolabelled pendimethalin (phenyl-ring-u- ${ }^{14} \mathrm{C}$ labelled, specific activity $10.7 \mathrm{MBq} /$ mg, radiochemical purity $99.2 \%$, BASF SE) was used. Reference standards for pendimethalin metabolites 2,6-Dinitro-3,4-dimethylaniline (purity 96.1\%, “M455H032”), 1-(1-ethylpropyl)-5,6-dimethyl-7-nitro-1H-benzimidazole (purity 99.0\%, “M455H029”), 4,5-dimethyl-3-nitro-N2-(pentan-3-yl)benzene-1,2-diamine (purity 98\%, "M455H033") and 2-methyl-3,5-dinitro-4-(pentan-3ylamino)benzoic acid (purity 98.2\%, "M455H001") were provided by BASF SE to support the identification of the detected degradation products. For chemical structures see Additional file 1: Table S7.

As a control substance ${ }^{14} \mathrm{C}$-labelled sodium benzoate (phenyl-ring-u- ${ }^{14} \mathrm{C}$ labelled, specific activity $12.84 \mathrm{MBq} /$ mg, American Radiolabeled Chemicals) was used to prove the viability of the aqueous system as proposed by OECD 309 [1].

\section{Test system}

\section{Surface water}

The study was conducted using Hennesee lake water, an artificial fresh water lake in Germany $51^{\circ} 19^{\prime} 57^{\prime \prime} \mathrm{N}, 8^{\circ} 15^{\prime}$ $48^{\prime \prime} \mathrm{O}$, with woodland around the lake. The water system was sampled from Hennesee lake on December 1st, 2017. For details of the sampling see Additional file 1: Table S1. The key characteristics of the surface water are shown in Additional file 1: Table S2.

Local sediment was sampled at the same place and sieved $<2 \mathrm{~mm}$. The sediment was sampled frequently and data from previous years show no significant change of the sediment over time (see Additional file 1: Table S4).

To monitor the microbial activity of the sampled water, $250 \mathrm{~mL}$ from each vessel were transferred to an erlenmeyer type glass flasks with a capacity of $0.5 \mathrm{~L}$ and treated with ${ }^{14} \mathrm{C}$-sodium benzoate at a concentration of $12 \mu \mathrm{g} / \mathrm{L} / 38.5 \mathrm{kBq}$ per flask. The flasks were sealed with ground joint caps equipped with a gas inlet and outlet. The inlet was connected gas-tight to a gas supply, which continuously supplies moistened $\mathrm{CO}_{2}$-free air in order to ensure aerobic incubation conditions. The lids outlet was connected to two wash bottles filled with $2 \mathrm{M} \mathrm{NaOH}$ to trap ${ }^{14} \mathrm{CO}_{2}$ from mineralization of the sodium benzoate. At test end further water was taken from the $900 \mathrm{~L}$ test vessels and tested again as described above.

\section{Test setup and incubation}

The test setup consisted of 4 test vessels with a size of about $1000 \mathrm{~L}$ each. They were made of stainless steel in the form of a vertically cut cylinder. The height of the cylinder was $140 \mathrm{~cm}$ and the surface are was about $0.7 \mathrm{~m}^{2}$. The test vessels were placed in temperature controlled containers with two test vessels per container. The space in between container and test vessel was filled with water. The test temperature in the container was set to $20^{\circ} \pm 2{ }^{\circ} \mathrm{C}$. It was placed on weighing scales, which allowed control of the volume to a precision of $1 \mathrm{~L}$.

$900 \mathrm{~L}$ of the test water were pumped through a $100 \mu \mathrm{m}$ mesh filter directly into each of the test vessels. Two vessels, one in each container, were additionally applied with $900 \mathrm{~g}$ sediment dry matter, corresponding to $1 \mathrm{~g}$ dry matter per liter. As the vessels were not stirred or shaken, the sediment settled and on the application day the water was almost clear again.

The test vessels in one container were covered with a stainless steel lid to protect from light. The two vessels in the other container were covered with a lid of borosilicate glass and exposed continuously to simulated sunlight to study the influence of sunlight on the fate of pendimethalin. The light source was an Osram HQI-TS $2000 \mathrm{~W}$ metal halide lamp. Light spectrum and -intensity were measured by a UV/VIS spectrometer (BLACK-Comet, Stellarnet) and evaluated with the software SpectraWiz. The sunlight spectrum as well as sunlight intensity $\left(633 \mathrm{~W} / \mathrm{m}^{2}\right)$ were measured at noon on July 18th 2017 with the same instrumentation. The lamp was installed at $200 \mathrm{~cm}$ distance from the water surface, the light intensity measured at the center of the water surface at test start was $1222 \mathrm{~W} / \mathrm{m}^{2}$. The light spectrum at the water surface under the glass lid showed a wavelength cut off at $290 \mathrm{~nm}$ (see Additional file 1: Fig. S2). The spectrum was comparable to natural sunlight between 290 to $800 \mathrm{~nm}$ with the exception of a peak at $535 \mathrm{~nm}$ which is caused by the metal halide lamp (see Additional file 1: Fig. S1). Since pendimethalin shows no absorbance at this wavelength (UV-spectrum pendimethalin see $[12,13]$ ) this emission peak is considered not relevant for the test.

So finally, four different test setups were prepared: (a) water without sediment (pelagic) with sunlight simulation (PS), (b) water with suspended sediment with 
sunlight simulation (SSS), (c) water without sediment in the dark (PD) and d) water with suspended sediment in the dark (SSD). All test vessels were incubated at $20{ }^{\circ} \mathrm{C}$ for 60 days.

\section{Sampling}

In order to avoid water losses by evaporation the test vessels exposed to simulated sunlight were covered by a lid of borosilicatglass. The space between the water surface and the borosilicatglass lid was connected to wash bottles which were filled with ethylene glycol, $1 \mathrm{M} \mathrm{H}_{2} \mathrm{SO}_{4}$ and $2 \mathrm{M} \mathrm{NaOH}$, respectively. The gas in the headspace was permanently sucked through the wash bottles in order to trap volatile degradation products and ${ }^{14} \mathrm{CO}_{2}$. Gas trapping from the dark vessels was performed in the same way as described for the irradiated vessel.

Sampling was performed at 5 different water depths with minimum disturbance of the water body in order to avoid mixing the layers. For this purpose five 5 stainless steel tubes with an outer diameter of $8 \mathrm{~mm}$ but of different length were installed in each test vessel. Through the tubes at depths of $5 \mathrm{~cm}, 35 \mathrm{~cm}, 65 \mathrm{~cm}, 95 \mathrm{~cm}$ and $125 \mathrm{~cm}$ samples were taken by sucking water into bottles. The first $200 \mathrm{~mL}$ were discarded at each sampling. Figure 1 shows the sampling tubes before the container was filled with water.

The test was performed with a mixture of ${ }^{14} \mathrm{C}$-labelled pendimethalin and non-labelled pendimethalin at an initial pendimethalin concentration of about $94 \mu \mathrm{g} / \mathrm{L}$ (49 $\mathrm{MBq}$ per test vessel). Considering the surface area of the test vessel, the applied amount would correspond to a direct overspray of an agricultural use rate of $1.1 \mathrm{~kg}$ pendimethalin per ha. The test substance was applied dissolved in $4500 \mathrm{~mL}$ with acetonitrile which is equal to $0.5 \%$ solvent application per water volume of a test vessel. After application, the vessels were manually stirred with a $1 \mathrm{~m}$ stainless steel paddle to homogenously distribute the test substance in the water body. The concentration of pendimethalin measured directly after application in different water depths proved a homogenous distribution at test start (see Additional file 1: Table S5). Samples were taken at $0,1,3,7,10,14,22,30$ and 58 days after treatment from all vessels at the five sampling depths. Duplicate sampling was performed for each sample. At each sampling water parameters $\mathrm{pH}$, temperature, oxygen content and redox potential were measured in the test vessels.

\section{Sample processing}

To determine dissolved ${ }^{14} \mathrm{CO}_{2}$, an aliquot of each water sample was acidified with concentrated $\mathrm{HCl}$. The amount of ${ }^{14} \mathrm{CO}_{2}$ released was calculated from the difference of

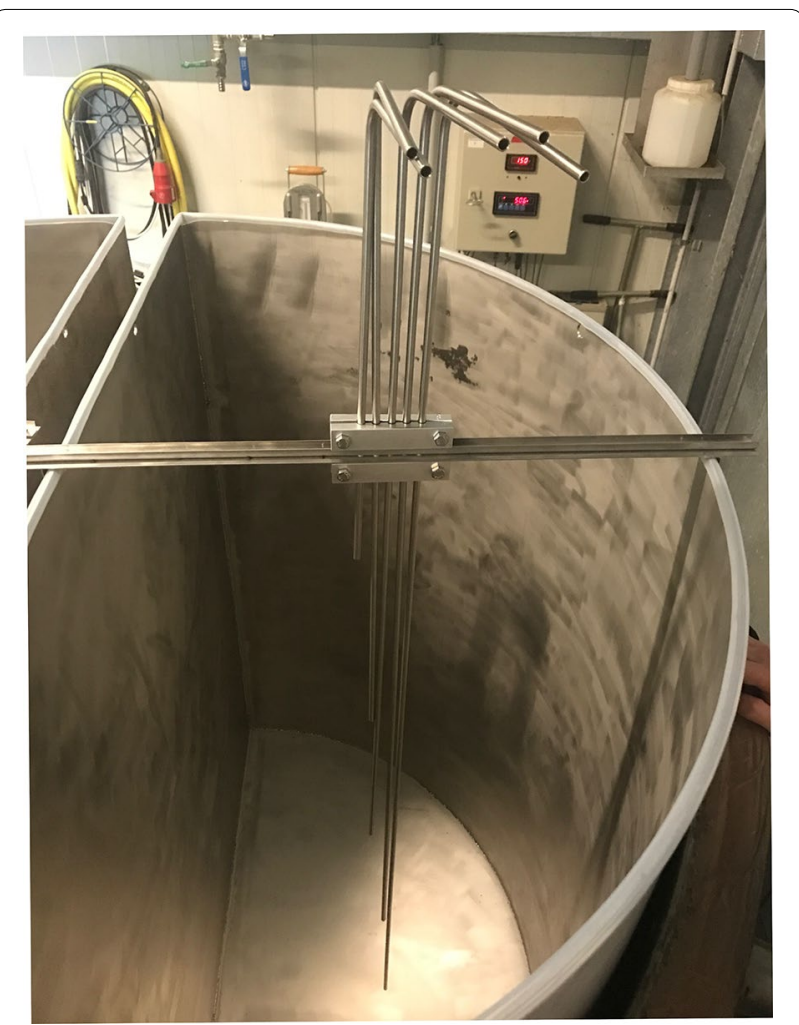

Fig. 1 Steel tubes for water sampling at different depths without disturbing the water body

radioactivity measured by LSC (liquid scintillation counting) before and after acidification of the sample.

Another aliquot of the water samples from the pelagic test setups were diluted 1:1 with methanol for stabilization. Samples from the suspended sediment setups were centrifuged $(28,000 \times g)$ before stabilization. Aliquots from the stabilized samples were measured by LSC for dissolved radioactivity.

Radioactivity in liquid samples was determined with Hidex Instruments 300SL liquid scintillation counters (LSC). $1 \mathrm{~mL}$ of the stabilised water samples was mixed with $4 \mathrm{~mL}$ of Ultima Gold (Perkin Elmer) as scintillator.

Quantification of pendimethalin and metabolites was done by radio-TLC and LC-MS. Pendimethalin was quantified by mass spectrometric analysis with a limit of detection (LOD) of $0.01 \mu \mathrm{g} / \mathrm{L}$ and a limit of quantitation (LOQ) of $0.05 \mu \mathrm{g} / \mathrm{L}$. Metabolites were identified by comparison with retention times of reference substances. Details of the analytical methods are given in the Additional file 1 .

\section{Kinetic evaluation}

Kinetic analysis and calculation of $\operatorname{DegT}_{50}$ and $\operatorname{DegT}_{90}$ values were performed with the kinetic evaluation tool 
CAKE, following the recommendations of the FOCUS kinetics working group [14]. CAKE version 1.4 (Release) running on $\mathrm{R}$ version 2.15.1 (2012-06-22) [6] was used.

Kinetic analysis and calculation of $\operatorname{DegT}_{50}$ and $\operatorname{Deg} \mathrm{T}_{90}$ values were performed for each setup individually. In a first step an average concentration was calculated for each test vessel since the concentration measured in the different depths varied. But for a kinetic calculation over the entire test vessel, one concentration is necessary to be calculated for each sampling time. In order to calculate the average concentration it was assumed that the concentration measured for each water depth is representative of a $30 \mathrm{~cm}$ water layer, in this case $15 \mathrm{~cm}$ above and $15 \mathrm{~cm}$ below the sampling depth. Since the uppermost sampling was carried out at a depth of only $5 \mathrm{~cm}$, this sample consequently only represents a total of a $20 \mathrm{~cm}$ water layer with $15 \mathrm{~cm}$ below but only $5 \mathrm{~cm}$ above the sampling depth. A water layer of $30 \mathrm{~cm}$ in a cylindric vessel of $140 \mathrm{~cm}$ hight represents the 30/140s part of the entire vessel. Accordingly for the averaging the concentration values from the different layers were weighted according to the part of the total volume that they represent. So a $30 \mathrm{~cm}$ layer was weighted with $30 / 140$ while the upper layer of $20 \mathrm{~cm}$ was weighted with 20/140 only. In total this result in one average concentration for the vessel. Kinetic calculations were performed based on the average vessel concentration calculated as described above.

\section{Results and discussion Test system parameters Viability of test water}

Viability controls by determination of the mineralization of ${ }^{14} \mathrm{C}$-sodium benzoate as reference standard were performed at test start and test end to monitor the microbial activity of the test waters as suggested in OECD 309 (see chapter 3.2 ). The viability control showed a mineralization of more than $50 \%$ within 14 days after test initiation, indicating an active microbial community in water directly after field sampling. At test end after 58 days, the viability check showed only a minor decrease of activity in the test water from the simulated sunlight setups, whereas activity in the test water from the dark setup decreased significantly. The water from the dark pelagic setup showed a mineralization of only $10 \%$ within 14 days which indicates a significant decrease of microbial activity.

At the same time a drop in oxygen concentration and redox potential in the dark setups was observed from 30 days onwards, accompanied by a strong anaerobic smell of the test water in the dark setups at test end and the water colour turned black (see Additional file 1: Fig. S3). Furthermore, on the surface of the water in the dark setups a gel-like substance had formed a closed layer of 1-2 cm thickness (see Additional file 1: Fig. S4).

In the setups with simulated sunlight neither visible change of the water nor an anaerobic smell was observed until test end. Oxygen content and redox potential slowly decreased but did not drop as observed in the dark setups. For details of water parameters see Additional file 1: Table S6.

\section{Simulated sunlight}

The light intensity was measured at each sampling depth up to the $125 \mathrm{~cm}$ water column directly in the test vessel. For the measurement, the Stellarnet sensor was attached to a rod and immersed in the water to the sampling depths. The filter cap, which is needed for measurements at this light intensity, protects the sensor from the water. The glass fibre connection from the sensor to the detector is waterproof. Results presented in Additional file 1: Fig. S5 show a significant decrease to about half the intensity at the $125 \mathrm{~cm}$ depth relative to the intensity measured at the water surface. The reason for this decrease was not analysed in further detail.

\section{Temperature}

The water temperature of $20{ }^{\circ} \mathrm{C} \pm 2{ }^{\circ} \mathrm{C}$ could only be maintained in the dark setup. In the vessels with sunlight simulation the temperature measured in $5 \mathrm{~cm}$ water depth ranged between $27^{\circ}$ and $28{ }^{\circ} \mathrm{C}$. This is caused by the sunlight simulation that, beside light, introduced also a lot of thermal energy. In the absence of mechanical mixing of the water body, the temperature of the upper water layer increased to $28^{\circ} \mathrm{C}$. In order to record the vertical temperature profile, the temperature was measured in $10 \mathrm{~cm}$ steps up to $130 \mathrm{~cm}$. Figure $S 6$ in Additional file 1 shows that the elevated temperature was limited to the upper $20 \mathrm{~cm}$ of the test vessel. The temperature in the water body below $20 \mathrm{~cm}$ was homogenously $20^{\circ} \mathrm{C} \pm 2{ }^{\circ} \mathrm{C}$.

\section{Distribution of radioactivity and mass balance}

The recovered radioactivity in all setups ranged from $73.4 \%$ AR (applied radioactivity) to $106.3 \%$ AR. The results of simulated sunlight and dark setup showed significant differences.

In the simulated sunlight setups a trend to decreasing recoveries with time was observed. The radioactivity dissolved in the water phase after acidification decreased from $100 \%$ at test start to just $50 \%$ after 58 days. On the other hand, dissolved ${ }^{14} \mathrm{CO}_{2}$ released by acidification, increased up to $22 \% \mathrm{AR}$ at test end indicating mineralization of pendimethalin. This might be a reason for the low recovery from sunlight simulation setups as that level of mineralisation was unexpected and the sampling procedure was not specifically designed to keep 
${ }^{14} \mathrm{CO}_{2}$ dissolved in the water sample. Losses might have occurred while sucking the water by vacuum in the sampling bottles. In the volatile traps connected to the test vessels less than $0.4 \%$ AR was collected in any of the setups, no pendimethalin was detected. So, ${ }^{14} \mathrm{CO}_{2}$ produced by mineralization stayed dissolved in the water phase.

However, losses of volatiles, in particular ${ }^{14} \mathrm{CO}_{2}$, cannot be avoided completely in simulation systems of about $1 \mathrm{~m}^{3}$ size. This is supported by the results from the dark setups, where recovery was much better after 58 days. Some variations were observed during incubation but without a clear trend. The recovered radioactivity was almost entirely detected in the water after acidification. Neither dissolved ${ }^{14} \mathrm{CO}_{2}$ nor significant amounts of radioactivity in the volatile traps were found at any time. This is a significant difference to the setups with simulated sunlight exposure, where relevant mineralization of pendimethalin was observed.

The sediment from the suspended sediment setups was sampled only at test end. Amounts of 4.4\% (SSS) and 7.7\% AR (SSD) were found in the sediment. This is in line with the sorption coefficient [12], which theoretically estimates an amount of 3 to $9 \%$ AR in the sediment in the given system and indicates a more or less homogeneous distribution of pendimethalin in the test systems at the end of the study duration.

\section{Degradation behaviour of pendimethalin}

Quantification of parent pendimethalin was based on LC-MS analysis. In the simulated sunlight setups the pendimethalin concentration reduced within 30 days to less than $2 \%$ of the initially applied concentration. The samples from different water depths were very similar in concentration. This is not surprising as there was permanent exchange between the water layers by diffusion.

In the dark setups degradation was slower compared to the simulated sunlight setups. Independent of the decrease of microbial activity and oxygen concentration, a slow degradation of pendimethalin was expected from the results of the standard OECD 309 laboratory test, which was also conducted in dark conditions [12]. In the SSD setup, the pendimethalin concentration varies through the water layers but the overall degradation was faster than in the dark pelagic setup. The concentration at day 58 in the SSD setup decreased below $1 \%$ of the initial concentration while in the PD experiment $21 \%$ of the initially applied pendimethalin was still present.

\section{Degradation profile of pendimethalin under different incubation conditions}

Radio-TLC were used for metabolic profiling due to its analytical sensitivity and potential to detect also unknown metabolites where no reference standards are available. Pendimethalin could not be separated chromatographically from the metabolites M455H029 and M455H033 (see “Test and reference substances" section). Thus, the signal at this Rf represent the sum of at least those three substances. The metabolites M455H001 and M455H032 were separated by radio-TLC.

The metabolic degradation profiles of pendimethalin differ significantly in the simulated sunlight and dark setups. In both dark setups the pendimethalin concentration detected by radio-TLC matches very good with the values determined by LC-MS. This indicates on the other hand that no significant amounts of metabolites M455H029 and M455H033 were present in the samples as this would have increased the radio-TLC-signal of pendimethalin by co-elution.

M455H001 was found only at day 58 with $4.7 \%$ and $6 \%$ AR (average values over all water layers), M455H032 was found only in the PD setup with $8 \%$ AR but not in the SSD setup. Total unidentified radioactivity, e.g. where the Rf-value could not be assigned to one of the available reference standards, became significant at day 30 and increased until day 58 to $44.6 \%$ AR in the PD setup. In the SSD setup this fraction was already present at day 10 and increased to $67 \%$ AR at day 58 .

In the simulated sunlight setups the pendimethalin amounts determined by radio-TLC match with the LCMS measurements, except for day 7 and day 10. At these days, radio-TLC detected up to $28 \%$ more radioactivity at the pendimethalin $\mathrm{Rf}$ than expected according to LCMS measurement. This was probably due to metabolites M455H029 and M455H033 being present in the samples in which case they would co-elute with pendimethalin and increase radioactivity at this retention time.

The sample from day 14 was not considered as those data from LC-MS were already classified as outliers (see Additional file 1: Table S8) which was confirmed by the radio-TLC analyses.

M455H001 was found only in minor amounts up to 5\% AR in certain samples. M455H032 was detected from day 3 (SSS) and day 7 (PS). It increased until day 14 to $13.8 \%$ AR and $14.7 \%$ AR and then decreased to $0 \%$ and $4 \%$ AR at day 58. Total unidentified radioactivity appeared at day 3 in both setups and increased to a maximum of $36.7 \%$ at day 22 (SSS) and 43.7\% AR at day 30 (PS) before it decreased slowly.

\section{Relevance of direct and indirect photolysis}

Metabolite M455H032 is described in the literature [12, 15] as photolysis product of pendimethalin. This is confirmed by the present study as it was detected in the simulated sunlight setups only. Decreasing amounts after day 14 prove that M455H032 was further degraded under the test conditions. 
M455H033 is also a known photolysis product of pendimethalin [15]. If M455H032 was found, M455H033 should have been detected as well. Unfortunately there is no direct proof since M455H033 co-eluted with parent pendimethalin on radio-TLC. Nevertheless, as described above, deviations between pendimethalin determined by LC-MS and pendimethalin detected by radio-TLC, were observed only in the simulated sunlight setups for day 7 and day 10 . These are the samples were also M455H032 was detected and it is assumed that the observed deviation might be due to co-eluting M455H033. At least it is a further indication that photolysis was a relevant process for the fate of pendimethalin in the simulated sunlight setups.

In both simulated sunlight experiments, maximum amounts of M455H032 were always determined in the top $5 \mathrm{~cm}$ water layer. Since this was the case from more than 40 individual samples this is a further indication that M455H032 is a photolysis product because the irradiation energy was highest in the top water layer. However, it was also found in deeper water layers. That makes sense because as shown in Additional file 1: Fig. S5 the irradiation energy in the water body did not decrease to zero until the $125 \mathrm{~cm}$ depth and thus photolysis products should be detectable in all water layers. Indirect photolysis might have been a relevant process as well. Dissolved salts and humic substances might have acted as sensitizers to further increase photolytic degradation $[9,10]$. However, the actual experimental setup was not capable to distinguish between direct and indirect photolysis.

A further result of simulated sunlight application is the significantly increased mineralization of pendimethalin (see "Distribution of radioactivity and mass balance" section) whereas in both dark setups no mineralization was observed up to day 58 .

\section{Kinetic evaluation \\ Averaged concentrations for the different experimental setups}

Table 1 shows the calculated average data used for kinetic evaluation. For the PS setup one outlier was identified at day 14 and corresponding data were not considered for any kinetic evaluation. The degradation plots are shown in Fig. 2.

The test setups with exposure to simulated sunlight showed a significant faster degradation of parent pendimethalin compared to the dark setups. Detection of known photolysis products of pendimethalin in the simulated sunlight setups and absence of those products in the dark setups are further evidence for the relevance of photolysis processes for the fate of pendimethalin under the applied conditions. Application of $1 \%$ sediment to the test systems further increased the degradation rate. The
Table 1 Pendimethalin concentration (weighted average) in $\mu \mathrm{g} / \mathrm{L}$ used for kinetic evaluation

\begin{tabular}{lcrrr}
\hline Time (days) & \multicolumn{1}{c}{ PS } & \multicolumn{1}{c}{ PD } & \multicolumn{1}{c}{ SSS } & \multicolumn{1}{c}{ SSD } \\
\hline 0 & 101.7 & 100.3 & 99.9 & 100.1 \\
1 & 98.6 & 99.6 & 102.4 & 105.5 \\
3 & 87.0 & 96.8 & 77.8 & 89.4 \\
7 & 64.8 & 102.0 & 52.6 & 69.6 \\
10 & 54.9 & 86.3 & 38.0 & 76.5 \\
14 & $71.2^{*}$ & 72.6 & 43.6 & 72.2 \\
22 & 6.4 & 62.5 & 4.9 & 42.0 \\
30 & 1.0 & 51.6 & 1.0 & 13.4 \\
58 & 0.1 & 21.3 & 0.0 & 0.1 \\
\hline
\end{tabular}

${ }^{*}$ Classified outlier, not considered for kinetic evaluation

degradation endpoints determined based on the average system concentrations are presented in Table 2 . The half-lives presented in Table 2 are valid for the experimental setups only. If transferred to certain field and sunlight conditions, the irradiation energy of the simulated sunlight and the applied continuous irradiation, without day/night rhythm, have to be taken into account in case of recalculations considering specific outdoor light scenarios.

\section{Influence of sampling depths on degradation kinetics}

Degradation kinetics were also calculated for the concentration curve of each individual sampling depth, though it was understood that the water layers are not independent but connected systems with the potential of substance exchange e.g. by diffusion. Nevertheless, the evaluation shows further differences between the test setups.

In the dark setups no trend for depths dependent degradation kinetics was observed. Kinetic behaviour was similar at all sampling depths.

In the PS setup only minor differences were observed in the different layers. The degradation in the top layer was slightly faster than in the other layers. The same could be observed in the SSS setup, but with significant faster degradation in the upper layer (see Additional file 1: Table S8). The results of the calculated best-fit $\operatorname{DegT}_{50}$ and $\operatorname{DegT}_{90}$ values for pendimethalin (SFOkinetic model) are shown in Table 3.

Potential reasons for the faster disappearance in the upper layer are the simulated sunlight and the increased temperature of that water layer. As described above there is a strong indication of relevant photolysis in the SSS setups. The photolysis product M455H032 has been identified and the maximum concentration has been found in the upper water layer, where light intensity was highest. This would explain the higher dissipation rate 

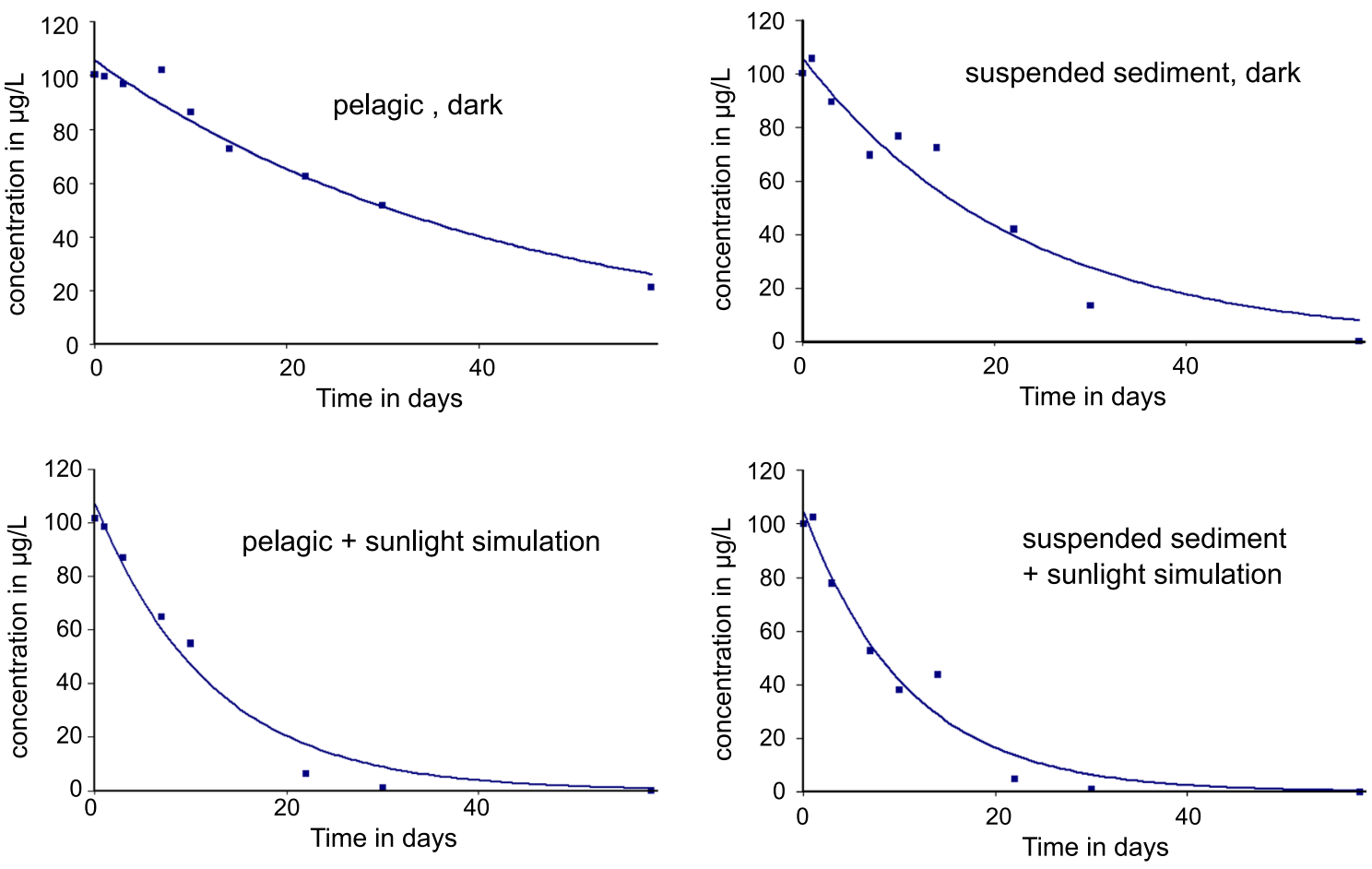

- Observations

Fit

Fig. 2 Single first order degradation kinetics of pendimethalin under different incubation conditions (weigthed mean over all water layers, evaluation with CAKE [17])

Table 2 Average degradation kinetics (best fit: SFO-kinetics, CAKE evaluation tool) across the test setups

\begin{tabular}{lllll}
\hline Test setup & DegT $_{\mathbf{5 0}}$ (days) & DegT $_{\mathbf{9 0}}$ (days) & Coefficient of determination $\left(\boldsymbol{r}^{\mathbf{2}}\right)$ & Chi $^{\mathbf{2}}$ error $(\%)$ \\
\hline PS & 8.38 & 27.84 & 0.9795 & 9.613 \\
SSS & 7.52 & 24.97 & 0.9684 & 11.72 \\
PD & 28.83 & 95.77 & 0.9603 & 5.448 \\
SSD & 15.52 & 51.54 & 0.9389 & 11.27 \\
\hline
\end{tabular}

Table 3 degradation kinetics (SFO-kinetic, CAKE model) for different sampling depths at SSS setup

\begin{tabular}{lllll}
\hline Test setup: SSS $(\mathbf{c m})$ & Dis $^{*} \mathbf{T}_{\mathbf{5 0}}$ (days) & Dis*$^{*} \mathbf{T}_{\mathbf{9 0}}$ (days) & Coefficient of determination $\left(\boldsymbol{r}^{\mathbf{2}}\right)$ & Chi $^{\mathbf{2}}$ error $(\%)$ \\
\hline 5 & 5.789 & 19.23 & 0.9685 & 13.11 \\
35 & 7.174 & 23.83 & 0.9683 & 10.42 \\
65 & 7.616 & 25.3 & 0.965 & 10.49 \\
95 & 8.318 & 27.63 & 0.9566 & 13.13 \\
125 & 8.179 & 27.17 & 0.9443 & 12.55 \\
\hline
\end{tabular}

*"Dis" instead of "Deg" because substance exchange between the layers is possible

of pendimethalin in the upper water layer but does not allow a numerical estimation of the influence of the degradation kinetics.
The influence of the elevated temperature can be estimated by the Q10 factor [16]. Q10 is the factor of the increase of the degradation rate at an increase of the temperature by $10{ }^{\circ} \mathrm{C}$. It has been derived for soil degradation 
studies but is in principle a physical parameter and can serve as a rough estimate also in water. In the current experiment the temperature in the upper layer was measured to be almost $28{ }^{\circ} \mathrm{C}$ on the water surface with linear decrease to $20{ }^{\circ} \mathrm{C}$ at a depth of $20 \mathrm{~cm}$ (see Additional file 1: Fig. S6). Thus, the average temperature in the top $20 \mathrm{~cm}$ can be estimated to $24{ }^{\circ} \mathrm{C}$ which is $4{ }^{\circ} \mathrm{C}$ above the lower water layers. Following the Q10 theory, the factor Q4 has to be calculated by $2.58(4 / 10)=1.46$. That means that the half-life normalized to $20^{\circ} \mathrm{C}$ can be estimated by

$$
\begin{aligned}
\operatorname{DegT}_{50} & =5.789 \text { days }\left(\text { at } 24{ }^{\circ} \mathrm{C}\right) \times 1.46 \\
& =8.45 \text { days }\left(\text { at } 20^{\circ} \mathrm{C}\right)
\end{aligned}
$$

This estimated half-life fits well to the degradation kinetics determined for the lower water layers. However, since the water layers were not independent systems, the reason for the increased degradation kinetics is assumed to be a mixture of the effects described above.

\section{Conclusions}

From the results obtained it can be concluded that pendimethalin degraded rapidly in a water-sediment environment and also in surface water without sediment. Application of simulated sunlight was a highly important factor for the degradation rate and route of pendimethalin and also for the test system stability.

Decreasing light intensity in deeper water layers resulted in slightly slower degradation rates of pendimethalin. However, although metabolites from pendimethalin photolysis were detected at maximum concentration in the upper water layer, they were found in all water layers sampled. This indicated that photolysis was a relevant process for the fate of pendimethalin within the entire $140 \mathrm{~cm}$ water body. The relevance of simulated sunlight is supported by the fact that in the simultaneously conducted dark setups a lower dissipation rate in the upper water layer was observed and no photolysis products were detected at all. Further, with simulated sunlight considerable mineralization of pendimethalin was observed while no mineralization was found in dark setups at any time. The results confirm the non-persistence of pendimethalin in aerobic surface water under the influence of sunlight since the half-life of pendimethalin was clearly below the ECHA persistence trigger of 40 days for water [8].

For a substance that is known to be sensitive to aqueous photolysis like pendimethalin, results from a surface water degradation test under the influence of simulated sunlight are very different from those obtained from the same experiment but under dark conditions. This was proven in the current study and holds true for the degradation rate and degradation route. Standard TG OECD309, which represents the standard test guideline for "Surface Water-Simulation Biodegradation Test" allows exposure to "diffuse light" without any further definition of the light quality and thus prefers dark conditions. As OECD 309 is the relevant test guideline for persistency assessment in surface water under $\mathrm{REACH}$, this leads to an overestimation of persistency of hydrolytically stable, but photolytically sensitive substances in the overall PBT assessment for chemicals [8].

The study further showed that simulated sunlight helps to keep the test system stable and aerobic. Formation of a jelly phase in the dark test system is also frequently observed in the usual laboratory setup in standard OECD 309 testing. Although it is not studied so far what influence the jelly phase might have on substance degradation (e.g. by formation of non-extractable-residues).

\section{Supplementary information}

Supplementary information accompanies this paper at https://doi. org/10.1186/s12302-020-00402-w.

Additional file 1. Supplementary tables and figures and details on chemical analysis.

\section{Abbreviations \\ \% AR: \% Of applied radioactivity; Bq: Becquerel; DAT: Days after treatment; DegT $_{50 / 90}$ :Time where 50/90\% of the test substance is degraded; FOCUS: FOrum for the Co-ordination of pesticide fate models and their USe; GLP: Good Laboratory Practice; LC-MS: Liquid chromatography with mass spectrometric detection; LSC: Liquid Scintillation Counting; PD: Pelagic, dark; PS: Pelagic, sunlight simulation; SFO: Single first order; SSD: Suspended sediment, dark; SSS: Suspended sediment, sunlight simulation; TLC: Tin layer chromatography.}

\section{Acknowledgements}

The authors would like to thank BASF SE for supporting the present work.

\section{Authors' contributions}

$\mathrm{DH}$ had project lead and organization. MK lead the experimental work in the laboratory and data evaluation. JK performed the kinetic evaluation. JH was responsible for conceptual design of the project. $\mathrm{DH}$ and $\mathrm{JH}$ drafted the manuscript. All authors read and approved the final manuscript.

Funding

The study was funded by BASF SE. Open Access funding enabled and organized by Projekt DEAL.

Ethics approval and consent to participate Not applicable.

\section{Consent for publication}

Informed consent was obtained from all individual participants included in the study.

\section{Competing interests}

The authors declare that they have no competing interests. 


\section{Author details}

${ }^{1}$ Fraunhofer Institute for Molecular Biology and Applied Ecology, Auf dem Aberg 1, 57392 Schmallenberg, Germany. ${ }^{2}$ BASF SE, Crop Protection - Environmental Fate, Speyerer Strasse 2, 67117 Limburgerhof, Germany.

Received: 15 May 2020 Accepted: 14 September 2020

Published online: 02 October 2020

\section{References}

1. OECD Test Guideline 309: Aerobic Mineralisation in Surface Water-Simulation Biodegradation Test, OECD, adopted 13th April 2004

2. Völkel, W.; Höger, S.J. (2015): Aerobic Mineralisation in Surface Water (OECD 309): Experiences and Interpretation. Poster presentation SETAC Europe Barcelona 2015

3. Shrestha P, Junker T, Fenner K, Hahn S, Honti M, Bakkour R, Diaz C, Hennecke D (2016) Simulation studies to explore biodegradation in watersediment systems: from OECD 308 to OECD 309. Environ Sci Technol. https://doi.org/10.1021/acs.est.6b01095

4. Workshop on identifying limitations of the OECD water-sediment test (OECD 308) and developing suitable alternatives to assess persistence, 6 October 2015, Dübendorf (Switzerland)

5. Baena-Nogueras RM, González-Mazo E, Lara-Martín PA (2017) Degradation kinetics of pharmaceuticals and personal care products in surface waters: photolysis vs biodegradation. Sci Total Environ 590-591:643-654

6. Prasse C, Wenk J, Jasper JT, Ternes TA, Sedlak DL (2015) Co-occurrence of photochemical and microbiological transformation processes in openwater unit process wetlands. Environ Sci Technol 49(24):14136-14145

7. Rúa-Gómez PC, Püttmann W (2013) Degradation of lidocaine, tramadol, venlafaxine and the metabolites $\mathrm{O}$-desmethyltramadol and $\mathrm{O}$-desmethylvenlafaxine in surface waters. Chemosphere 90(6):1952-1959

8. ECHA Guidance on Infofmation Requirements and Chemical Safety Assessment, Chapter R.11: PBT/vPvP assessment, November 2014.
9. OECD Guideline for testing of chemicals, Proposal for a new guideline "Phototransformation of Chemicals in Water-Direct and Indirect Photolysis", Draft document, August 2000

10. OPPTS 835.5270: EPA Fate, Transport and Transformation Test Guidelines "Indirect Photolysis Screening Test", EPA712-C-98-099, January 1998

11. Castro-Jiménez, J, Van de meent. Accounting for photodegradation in P-assessment of chemicals, Reports Environmental Science no 381 Department of Environmental Science, Faculty of Science, Radboud University, 2011 https://doi.org/10.13140/rg.2.1.3597.8401.

12. EFSA (European Food Safety Authority) (2016) Conclusion on the peer review of the pesticide risk assessment of the active substance pendimethalin. EFSA J 14(3):4420. https://doi.org/10.2903/j.efsa.2016.4420

13. Perez ER, Le Calve S, Mirabel P (2008) Near-UV molar absortivities of alachlor, mecroprop-p, pendimethalin, propanil and trifluralin in methanol. J Photochem Photobiol A Chem. 193:237-244

14. FOCUS (2006) "Guidance Document on Estimating Persistence and Degradation Kinetics from Environmental Fate Studies on Pesticides in EU Registration" Report of the FOCUS Work Group on Degradation Kinetics, EC Document Reference Sanco/10058/2005 version 2.0, 434 pp

15. Roberts T (1998) Metabolic pathways of agrochemicals. Part one: Herbicides and plant growth regulators. The royal society of chemistry, London. ISBN 0.-85404-0494-9

16. EFSA (2007) Opinion on a request from EFSA related to the default Q10 value used to describe the temperature effect on transformation rates of pesticides in soil. EFSA J 2007(622):1-32

17. Report generated by CAKE version 1.4 (Release) CAKE developed by Tessella Plc, Abingdon, Oxfordshire, UK for Syngenta Running on.Net version 2.0.50727.8806

\section{Publisher's Note}

Springer Nature remains neutral with regard to jurisdictional claims in published maps and institutional affiliations.

\section{Submit your manuscript to a SpringerOpen ${ }^{\circ}$ journal and benefit from:}

- Convenient online submission

- Rigorous peer review

- Open access: articles freely available online

- High visibility within the field

- Retaining the copyright to your article

Submit your next manuscript at $\boldsymbol{\nabla}$ springeropen.com 